\title{
Design and Simulation of the First Joint for a Prospective 5 Degrees of Freedom Manipulator with MR Actuators*
}

\author{
Sergey Pisetskiy \\ Department of Electrical and Computer Engineering \\ The University of Western Ontario \\ London, ON, Canada \\ Email: spisetsk@uwo.ca
}

\begin{abstract}
This paper presents the mechanical design and mathematical modeling for the first joint of a five degrees of freedom collaborative manipulator. The robot is actuated using Magneto-Rheological (MR) clutches in order to ensure full backdrivability and intrinsic torque control for safe human-friendly operation. One of the unique features of this design is the usage of a single motor for powering all five joints of the robot simultaneously and independently. After a brief description of the MR clutch principles, the details of the mechanical design and a state-space representation of the first joint are discussed. The paper will conclude with some preliminary simulation results.
\end{abstract}

\section{INTRODUCTION}

In recent years, the need for a human-friendly robot that can safely operate in close proximity to humans is driving progress in robotic technologies. Many research groups [4] [5] [6] [9] [10] achieved remarkable results in the development of automated systems that can interact with the person without posing a significant threat of injury.

Among all sources of danger, the risk of injury caused by a collision between a human and a robot is one of the biggest. Direct interactions make collisions unavoidable, and mitigating impacts from such collisions is one of the top priorities for research and development.

A number of approaches address the safety of robotic systems through development of compliant actuators. Possible solutions include variable stiffness transmission approach, elastic actuation, MR brakes and MR clutches, joint torque control, Distributed MacroMini Actuation, etc.

In this paper the Distributed Active Semi Active (DASA) approach [7] is briefly discussed, and the first steps in the development of an innovative five degrees of freedom (5-DOF) manipulator are presented.

DASA approach is intended to address the safety at the level of transmission, while the majority of commercially available

*This work was supported in part by Canad Foundation for Innovation (CFI) and Natural Sciences and Engineering Research Council (NSERC) of Canada under grant No.25031 and RGPIN-346166.

\author{
Mehrdad R. Kermani \\ Department of Electrical and Computer Engineering \\ The University of Western Ontario \\ London, ON, Canada \\ Email: mkermani@eng.uwo.ca
}

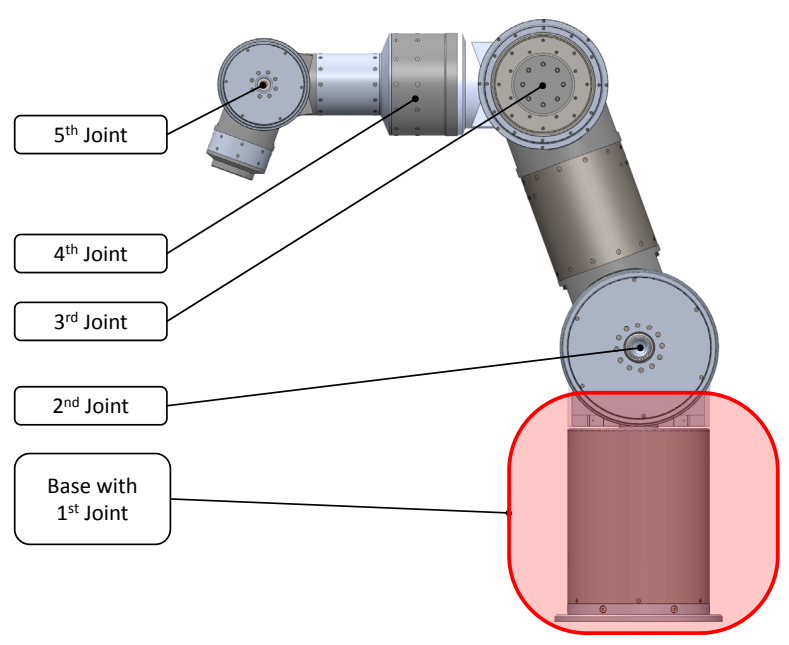

Fig. 1. Visualisation of the prospective 5-DOF robot.

robotics platforms (KUKA LBR iiwa, Kinova JACO, Franka Emika) utilize the joint torque control methodology, that could be subject to software errors or control system malfunction.

Rigorous studies regarding the safety of MR clutch for direct interactions with humans were presented previously [7]. This paper does not discuss and validate the safe characteristics of MR clutches for human-robot interactions.

The contributions of this work are as follows:

- An innovative mechanical design of the base of the intended 5-DOF manipulator is introduced. The base comprises a single motor that actuates all joints of the robot, a Harmonic Drive (HD) gearbox, and a pair of MR clutches for the first joint.

- $\quad$ The derivation of a state-space model of the first joint transmission is presented.

- $\quad$ The simulation results for a simple control algorithm and the developed mathematical model are discussed. 


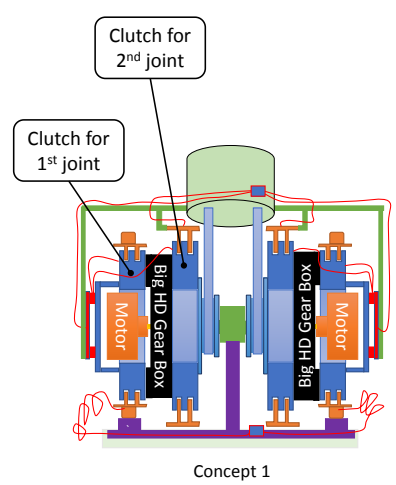

Concept 1

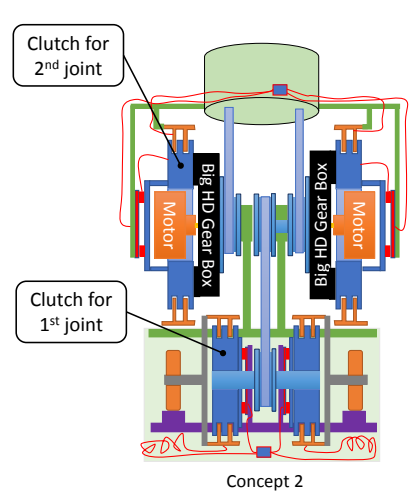

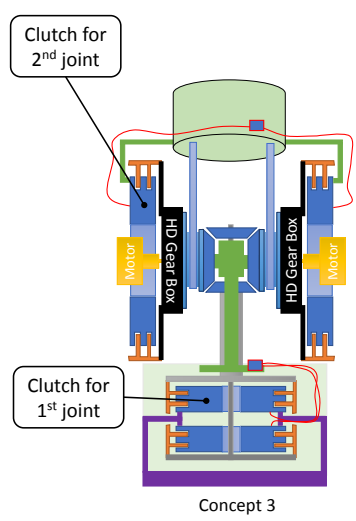

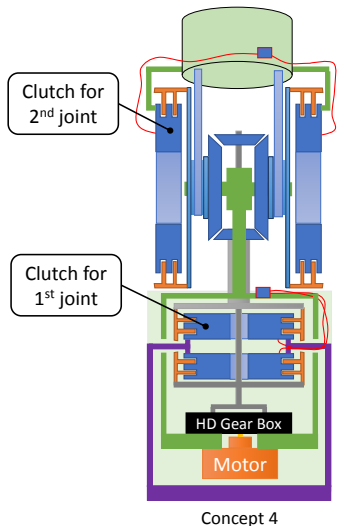

Fig. 2. Robot base design concepts

\section{OVERVIEW}

\section{A. Design Objective}

The main objective of the project is to develop a robotic manipulator using Magneto-Rheological actuators that address both safety and performance requirements of a collaborative robot. The manipulator includes five degrees of freedom that when combined with the degrees of freedom of the endeffector provide full kinematic redundancy. All joints of the intended manipulator are powered with the single motor located at the base of the robot. The targeted payload for the manipulator is $10 \mathrm{~kg}$ at full extension of the robotic arm.

Each joint of the manipulator is actuated using a pair of MR clutches coupled in an antagonistic configuration. The manipulator in total includes $10 \mathrm{MR}$ clutches and the torque is directly delivered by the clutches to the corresponding joint without gear reduction mechanism.

\section{B. Working Principles of MR clutches}

The utilization of a pair of MR clutches at each joint of the manipulator is a key feature of the proposed design. The main function of the MR clutch is the continual transmission of the actuation torque from the motor side to the load side. By changing the viscosity of the MR fluid in the clutch the exact amount of transmission torque between moving parts is regulated. The viscosity of MR fluid can be precisely controlled by applying a magnetic field in accordance with the requirement of the load transfer. The details of MR clutch construction and its features can be found in [2] [3] [8].

A pair of MR clutches can be configured to provide antagonistic actuation of a robot joint. In this case, the rotors of the MR clutches are rotated in opposite directions and the stators of both MR clutches are attached to a common shaft. Depending on the electromagnetic activation of each clutch, the torque can be applied by one or both MR clutches in either direction.

A complete comparison of various MR clutch configurations can be found in [1][2].

\section{Design of the Manipulator Base}

\section{A. Early design concepts}

At the early stage of this work, multiple design concepts for the base of the manipulator were considered and analyzed. In order to reduce the mass of the robot base, a lightweight high-speed motor with a harmonic drive gear box was decided to be used. Low weight along with a high gear ratio became the main reasons for choosing an expensive HD transmission for gear reduction.

Four concepts that were studied in details are shown in Fig. 2.

- The first concept comprises two motors and two pairs of clutches mounted co-axially on the wide moving frame. The two MR clutches of the 1st joint run on a circular rail. The motors are located inside the 1 st joint clutches, two harmonic drive gear boxes mounted between the 1 st and 2 nd joint clutches.

- The second concept is similar to the one discussed previously with the exception that the $2^{\text {nd }}$ joint clutches and the two motors and two harmonic drives are positioned above the $1^{\text {st }}$ joint clutches in order to reduce the diameter of the base.

- The third concept has a set of miter gears to change the orientation of the axis of rotation in order to mount $1^{\text {st }}$ joint clutches coaxial in the base. A pair of $2^{\text {nd }}$ joint clutches with a pair of motors and harmonic drives are positioned above the base.

- The fourth concept utilizes only one motor and one harmonic drive gear box. The pair of $1^{\text {st }}$ joint clutches is mounted coaxially to the vertical axis of rotation. The pair of $2^{\text {nd }}$ joint clutches is positioned above the base. A set of bevel gears distribute the motion between the clutches of the first and second joints.

The main disadvantage of the first concept is the unacceptable large size of the base and the need to use slip rings to actuate the magnetic coil and receive measurements from Hall sensors inside the MR clutches. Slip rings add additional 
resistance to the electrical circuit, bring more friction to the moving joint and may corrupt the signals of the sensors.

The second concept improves the base's footprint, but still requires slip rings to energize MR clutches and connect to Hall sensors.

The third and fourth concepts both have a small footprint in the base and do not require slip rings for MR clutches. But the third design requires two motors and two harmonic drives to ensure antagonistic actuation of the joints, while the last concept can use a single motor with a single HD gear box.

Taking into account the features described above, concept 4 was selected for further development.

\section{B. Transmission and control}

In selected configuration the single motor at the bottom of the robot base provides motion to the upper joints through a serial transmission that consists of several shafts and belts. As seen in Fig. 3, rotation speed of the motor shaft is reduced by the harmonic drive gear box.
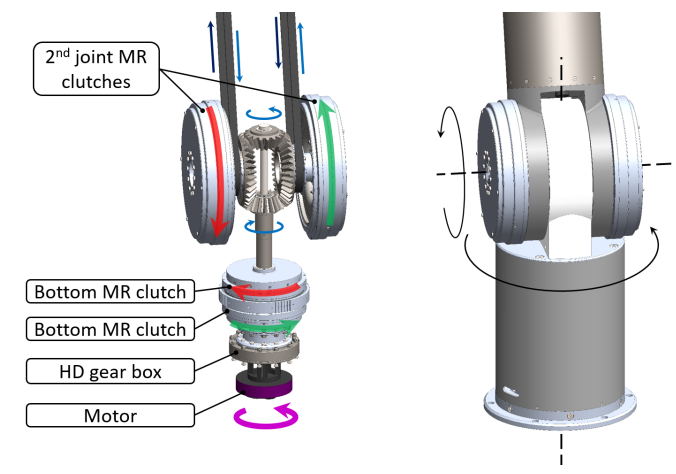

Fig. 3. Transmission of the 1-st and 2-nd joints of the manipulator.

The set of bevel gears located at the 2nd joint produce rotation in two opposite directions. Both clockwise and counterclockwise rotation is delivered to the pair of clutches at each joint through the belt transmission. Two belts moving in opposite directions provide antagonistic motion for the rotors in each pair of MR clutches.

\section{Design of the robot base (1-st joint)}

Manipulator base is designed to be properly mounted on the horizontal surface. The base comprises a pair of MR clutches that actuate the first joint responsible for the rotation of the second link around the vertical axis, as shown in Fig. 4. The single motor that drives all 5 upper joints is located at the very bottom in the base; it is fixed on the movable motor frame that rotates together with the upper links around the vertical axis of the base.

The wave generator of the harmonic drive gear box is directly connected to the motor shaft. The circular spline of the harmonic drive is mounted on the movable motor frame, while the flexspline is connected to the inner shaft and to the rotor of the bottom MR clutch via cylindrical adapters. The harmonic drive has high reduction ratio that allows utilization of the light but powerful high-speed electric motor.

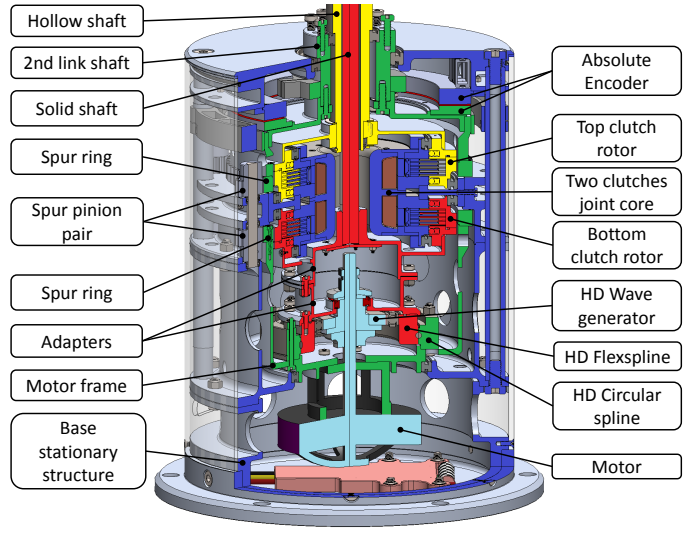

Fig. 4. Robot base zonal section view.

In order to compensate for the reaction moment, the torque from the motor and harmonic drive is transmitted from the motor frame to the upper links spur gears arrangement. The large spur gear - in the shape of a ring (spur gear ring) is located on the top of the motor frame. It is in mesh with six pinion pairs mounted on the stationary fixed cylindrical support. Through these pinion pairs, the rotation of the motor frame spur gear ring is mechanically transmitted to the similar spur gear ring that is mounted on the cylindrical frame of the second link. This construction allows to mechanically connect the motor frame with the second link frame, and at the same time provide fixation of the MR clutches to the stationary part of the base.

In the center of the manipulator base, there is a pair of MR clutches responsible for the rotation of the second robot link along the vertical axis (first joint). In order to save weight and space, both MR clutches share a joint magnetic core with two separate windings. Each clutch designed to generate torque up to $15 \mathrm{~N} \cdot \mathrm{m}$.

While the rotor of the bottom MR clutch is actuated directly by the circular spline of the harmonic drive, the rotor of the top clutch is rotated in opposite direction by the hollow shaft coming from the set of bevel gears located in the second link on top of the robot base.

In order to measure the angular position of the second joint, there is an absolute encoder mounted at the top plate of the manipulator base.

\section{Design of the 2nd joint}

The frame of the second link is mounted on the hollow shaft that spins around the vertical axis of the robot base. The link comprises a set of bevel gears to produce rotation in two opposite directions for all the upper joints of the robot. The transmission of the motion to the upper joints realized through the two belts as shown in Fig. 3.

From the harmonic drive located in the base, the rotation of the solid inner shaft is coming to the small bevel gear on the top of the link. Two big bevel gears in mesh with the small gear spin in opposite directions to each other with reduced speed 1:2. The bottom small bevel gear in mesh with big 
bevels rotates in an opposite direction relative to the top one. The hollow shaft coming from the bottom gear transmits the rotation to the rotor of the top MR clutch in the base.

Spinning in clockwise (CW) and counter-clockwise (CCW) directions big bevel gears rotate the rotors of two MR clutches of the second joint. The clutches should be able to produce up to $200 \mathrm{~N} \cdot \mathrm{m}$ torque each in order to actuate the third link of the robot.

\section{Modelling in MATLAB/Simulink}

\section{A. State-Space Model of the Joint}

In order to develop a state-space model, the simplified scheme of the manipulator first joint was considered, as shown in Fig. 5.

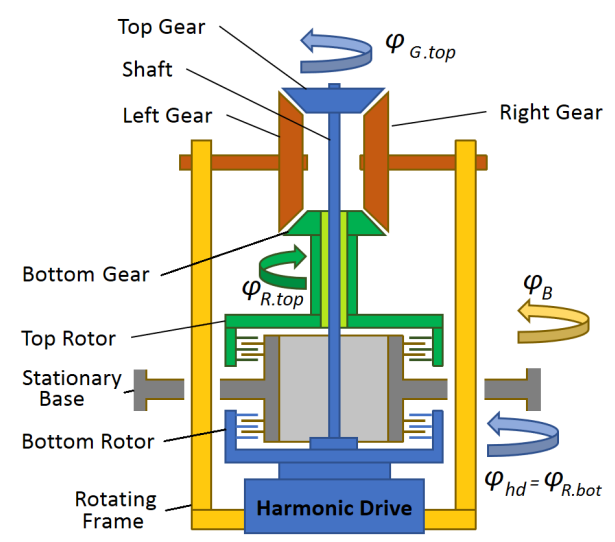

Fig. 5. Manipulator First Joint Scheme

In this figure, four separate groups of parts with common motions are identified with different colors, namely, HD Drive, bottom rotor, shaft, and top gear shown in blue; bottom gear, hollow shaft, and top rotor in green; rotating frame in yellow; and finally, left and right gears in red.

Assuming that the resulting angles, angular velocities, and angular accelerations of all parts of the assembly in the first group (blue color) are in the counterclockwise direction (looking from the top), then it can be shown that,

$T_{h d}-T_{R . b t m . i n e r t}-T_{R . b t m . f r}-T_{R . b t m . m r}-T_{\text {Sh.elast }}=0$

where $T_{h d}$ is the torque on Harmonic Drive, $T_{R \text {.btm.inert }}$ is the torque due to the inertia of the bottom rotor, $T_{R . b t m . f r}$ is the torque on clutch bottom rotor due to friction, $T_{R . b t m . m r}$ is the torque on bottom rotor due to MR effect, and $T_{\text {Sh.elast }}$ is the torque due to the elasticity of the shaft.

Harmonic drive output torque is a function of time,

$$
\begin{aligned}
T_{h d} & =T_{h d}(t) \\
T_{R . b t m . i n e r t} & =I_{R . b t m} \ddot{\phi}_{R . b t m}
\end{aligned}
$$

where $I_{R . b t m}$ and $\phi_{R . b t m}$ are the moment of inertia and the angle of the rotor of the bottom clutch respectively.

$$
T_{R . b t m . f r}=T_{R . b t m . v i s c}+T_{R . c o n s t . f r}
$$

where $T_{R . b t m . v i s c}$ is the torque on bottom rotor due to viscosity, $T_{R \text {.const.fr }}$ is the torque due to constant friction.

$$
T_{R . b t m . v i s c}=b_{R . v i s c} \dot{\phi}_{R . b t m}
$$

where $b_{\text {R.visc }}$ is the coefficient of viscous friction.

$$
\phi_{R . b t m}=\phi_{h d}
$$

where $\phi_{h d}$ is the angle of Harmonic Drive.

The torque due to the elasticity of the shaft can be calculated,

$$
T_{\text {Sh.elast }}=k_{S h} \Delta \phi=k_{s h}\left(\phi_{h d}-\phi_{G . t o p}\right)
$$

where $k_{s h}$ is the stiffness of the shaft, $\phi_{G . t o p}$ is the angle of the top bevel gear (top of the shaft).

For the second group of parts (green color), it is assumed that resulting angles, angular velocities, and angular accelerations of all parts of the assembly have clockwise direction (looking from the top), then it can be shown that,

$T_{G . b t m}-T_{R . t o p . i n e r t}-T_{R . t o p . f r}-T_{R . t o p . m r}-\frac{1}{2} T_{b . g . r e s u l t}=0$

where $T_{G . b t m}$ is the torque acting on the bottom bevel gear, $T_{R . t o p . i n e r t}$ is the torque due to the inertia of the clutch top rotor, $T_{R . t o p . f r}$ is the torque on clutch top rotor due to friction, $T_{R . t o p . m r}$ is the torque on clutch top rotor due to MR effect, $T_{\text {b.g.result }}$ is the resulting torque from bevel gears.

Due to mechanical differential properties, the torque acting on bottom gear is always equal to the top gear torque $T_{G \text {.top }}$,

$$
\begin{gathered}
T_{G . b t m}=T_{G . t o p}=T_{\text {Sh.elast }} \\
T_{\text {R.top.inert }}=I_{\text {R.top }} \ddot{\phi}_{\text {R.top }}
\end{gathered}
$$

where $I_{R . t o p}$ and $\phi_{R . t o p}$ are the moment of inertia and the angle of the rotor of the top clutch respectively.

$$
T_{R . t o p . f r}=T_{R . t o p . v i s c}+T_{R . c o n s t . f r}
$$

where $T_{R . t o p . v i s c}$ is the torque on bottom rotor due to viscosity. The top rotor rotates in opposite direction relative to the rotation of the stationary base.

$$
T_{\text {R.top.visc }}=b_{R . v i s c} \dot{\phi}_{R . t o p}
$$

where $\phi_{G . b t m}$ is the angle of the bottom bevel gear.

$$
\begin{gathered}
\phi_{\text {R.top }}=\phi_{G . b t m} \\
T_{\text {R.top.visc }}=b_{\text {R.visc }} \dot{\phi}_{G . b t m}
\end{gathered}
$$

In the third group (yellow color) the HD drive with the Motor is fixed on the rotating frame, so the reaction from the generated torque $T_{h d}$ is directly transmitted to the frame. Assuming that resulting angles, angular velocities, and angular accelerations of all parts of the frame assembly have counterclockwise direction (looking from the top),

$$
T_{\text {Sh.elast }}-T_{h d}+T_{G . b t m}-T_{F . \text { inert }}+T_{F}-T_{b . g . r e s u l t}=0
$$

where $T_{F \text {.inert }}$ is the torque due to the inertia of the rotating frame, $T_{F}$ is the torque acting on rotating frame from upper 
links (external). In this case torques $T_{\text {Sh.elast }}, T_{G . b t m}$, and $T_{F}$ have the same (counterclockwise) direction, while torques $T_{h d}$ and $T_{F . \text { inert }}$ have opposite direction.

$$
\begin{gathered}
T_{G . b t m}=T_{G . t o p}=T_{\text {Sh.elast }} \\
T_{\text {F.inert }}=I_{F} \ddot{\phi}_{F}
\end{gathered}
$$

where $I_{F}$ and $\phi_{F}$ are the moment of inertia and the angle of the rotating frame respectively.

In the fourth group (red color) the left and right bevel gears comprise the mechanical differential as well as transmit the torque from the top and bottom gears to the rotating frame.

According to design, both bevel gears have the same gear ratio $R_{b . g}$ in mesh with the top and with the bottom bevel gears. It is assumed that external torques applied to the left and right bevel gears have counterclockwise direction when looking from left and from right side respectively, so their resulting torque is:

$$
T_{b . g . r e s u l t}=R_{b . g} T_{G . L}+R_{b . g} T_{G . R}
$$

where $T_{G . L}$ and $T_{G . R}$ are the external torques acting on the left and right bevel gear respectively.

Angles of the mechanical differential in the rotating frame are governed by the following relation:

$$
\begin{gathered}
\phi_{G . t o p}+\left(-\phi_{G . b t m}\right)-2 \phi_{F}=0 \\
\phi_{G . b t m}=\phi_{G . t o p}-2 \phi_{F}
\end{gathered}
$$

In case of the motionless frame assembly, the angle $\phi_{G . b t m}$ has opposite direction relative to $\phi_{G \text {.top }}$ due to the property of mechanical differential.

For angular velocities and accelerations,

$$
\begin{aligned}
& \dot{\phi}_{G . b t m}=\dot{\phi}_{G . t o p}-2 \dot{\phi}_{F} \\
& \ddot{\phi}_{G . b t m}=\ddot{\phi}_{G . t o p}-2 \ddot{\phi}_{F}
\end{aligned}
$$

\begin{tabular}{|c|c|}
\hline $\begin{array}{l}x_{1}=\phi_{h d}(t), \\
x_{2}=\dot{\phi}_{h d}(t), \\
x_{3}=\phi_{G . t o p}(t), \\
x_{4}=\dot{\phi}_{G . t o p}(t), \\
x_{5}=\phi_{F}(t), \\
x_{6}=\dot{\phi}_{F}(t), \\
T_{1}=T_{h d}(t), \\
T_{2}=T_{R . b t m . m r}(t), \\
T_{3}=T_{R . t o p . m r}(t), \\
T_{4}=T_{G . L}(t),\end{array}$ & $\begin{array}{l}T_{5}=T_{G . R}(t), \\
T_{6}=T_{F}(t), \\
T_{7}=T_{R . c o n s t . f r}, \\
I_{1}=I_{R . b t m}, \\
I_{2}=I_{R . t o p}, \\
I_{3}=I_{F}, \\
b=b_{R . v i s c}, \\
k=k_{s h}, \\
R=R_{b . g} .\end{array}$ \\
\hline
\end{tabular}

Using relations described above, the following system with state equations was derived:

$$
\begin{aligned}
& {\left[\begin{array}{c}
\dot{x}_{1} \\
\dot{x}_{2} \\
\dot{x}_{3} \\
\dot{x}_{4} \\
\dot{x}_{5} \\
\dot{x}_{6}
\end{array}\right]=\left[\begin{array}{cccccc}
0 & 1 & 0 & 0 & 0 & 0 \\
-\frac{k}{I_{1}} & -\frac{b}{I_{1}} & \frac{k}{I_{1}} & 0 & 0 & 0 \\
0 & 0 & 0 & 1 & 0 & 0 \\
\frac{k\left(4 I_{2}+I_{3}\right)}{I_{2} I_{3}} & 0 & -\frac{k\left(4 I_{2}+I_{3}\right)}{I_{2} I_{3}} & -\frac{b}{I_{2}} & 0 & \frac{2 b}{I_{2}} \\
0 & 0 & 0 & 0 & 0 & 1 \\
\frac{2 k}{I_{3}} & 0 & -\frac{2 k}{I_{3}} & 0 & 0 & 0
\end{array}\right]\left[\begin{array}{l}
x_{1} \\
x_{2} \\
x_{3} \\
x_{4} \\
x_{5} \\
x_{6}
\end{array}\right]} \\
& +\left[\begin{array}{ccccccc}
0 & 0 & 0 & 0 & 0 & 0 & 0 \\
\frac{1}{I_{1}} & -\frac{1}{I_{1}} & 0 & 0 & 0 & 0 & -\frac{1}{I_{1}} \\
0 & 0 & 0 & 0 & 0 & 0 & 0 \\
-\frac{2}{I_{3}} & 0 & -\frac{1}{I_{2}} & -\frac{3 R}{I_{3}} & -\frac{3 R}{I_{3}} & \frac{2}{I_{3}} & -\frac{1}{I_{2}} \\
0 & 0 & 0 & 0 & 0 & 0 & 0 \\
-\frac{1}{I_{3}} & 0 & 0 & -\frac{R}{I_{3}} & -\frac{R}{I_{3}} & \frac{1}{I_{3}} & 0
\end{array}\right]\left[\begin{array}{c}
T_{1} \\
T_{2} \\
T_{3} \\
T_{4} \\
T_{5} \\
T_{6} \\
T_{7}
\end{array}\right]
\end{aligned}
$$

where variables are:

\section{B. Verification of the model in MATLAB/Simulink software}

Based on the state-space system above, the MATLAB/Simulink model was developed and analyzed. Based on the preliminary SolidWorks model analysis, the following values of variables were used in simulation:

$k=550[\mathrm{~N} \cdot \mathrm{m} / \mathrm{rad}]$ - Elastic coefficient of the shaft,

$b=0.5[\mathrm{~N} \cdot \mathrm{m} \cdot \mathrm{sec} / \mathrm{rad}]$ - Viscous friction coefficient,

$I_{1}=0.012\left[\mathrm{~kg} \cdot \mathrm{m}^{2}\right]$ - Inertia moment of bottom rotor,

$I_{2}=0.010\left[\mathrm{~kg} \cdot \mathrm{m}^{2}\right]-$ Inertia moment of top rotor,

$I_{3}=0.064\left[\mathrm{~kg} \cdot \mathrm{m}^{2}\right]$ - Inertia moment of rotating frame.

To build a discrete time model, a minimum required sampling rate was estimated (Nyquist criterion) and the sampling period $T s=0.01 \mathrm{sec}$ was selected.

In order to verify the developed model, the HD drive torque, continuous friction torque, and top clutch MR torque were applied in a form of a step-function:

- $\quad$ At the time $t=5 \mathrm{sec}$ the HD drive started to generate torque $T_{h d}=1 \mathrm{~N} \cdot \mathrm{m}$, the constant friction torque $T_{R . b t m . f r}=0.1 \mathrm{~N} \cdot \mathrm{m}$ appeared in the bottom clutch, and the constant friction torque $T_{R . t o p . f r}=0.1 \mathrm{~N} \cdot \mathrm{m}$ appeared in the top clutch;

- $\quad$ At the time $t=150 \mathrm{sec}$ the top MR clutch started to generate torque $T_{R . t o p . m r}=0.1 \mathrm{~N} \cdot \mathrm{m}$.

Resulting states representing angle and angular velocity of the bottom rotor, top bevel gear, rotating base, and derived parameters for top rotor are shown in Fig. 6 and Fig. 7.
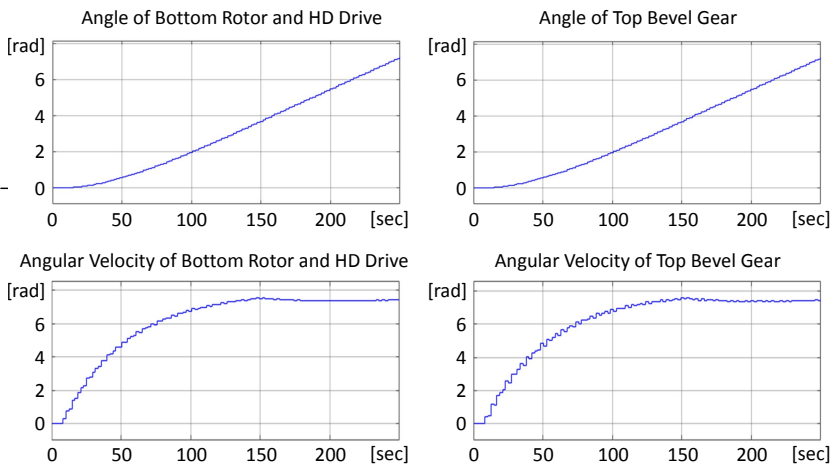

Fig. 6. Graphs of output angles and angular velocities for bottom rotor and top bevel gear

It can be observed on the graphs that at the time $=5 \mathrm{sec}$ when the harmonic drive started to generate torque $T_{h d}=1$ $N \cdot m$, angular velocities of the bottom rotor, top bevel gear, 

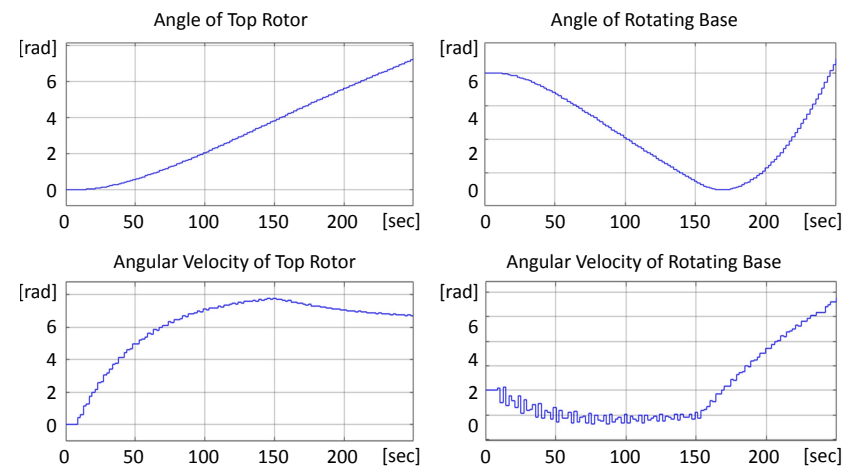

Fig. 7. Graphs of output angles and angular velocities for top rotor and rotating base gear

top rotor, and rotating base started to rise towards the certain values where the viscous friction of the parts equalizes generated torque. The rotating base started to turn in a clockwise direction and reached about $-0.07 \mathrm{rad}$ angle at the time $=150$ sec. On the angular velocity graphs for the top bevel gear and rotating base, one can clearly see the oscillations due to the flexibility of the shaft. Oscillations of the top and bottom rotor are not so visible but still can be noted.

When the top MR clutch was activated at $t=150 \mathrm{sec}$ and started to generate torque, the angular velocity of the top rotor started to decline, both bottom rotor and top bevel gear speeds showed a minor reduction, the angular velocity of the base changed its direction to counter-clockwise and the angle started to rise.

Shown on the graphs, the behavior of the parts corresponded very well with the expected motions of the actual hardware. Additional analogous simulations were performed to ensure the correct behavior of the developed system.

\section{Simple control design in MATLAB/Simulink software}

For further analysis, the simple input transducer is designed to process the desired reference trajectory of the rotating base into the controlling signals for clutches and HD drive. The required torques are calculated proportional to the derivative of the desired angle with respect to the time. The desired reference trajectory designed in MS Excel is shown with red color in Fig. 9.

The graphs of the torques applied to the system to follow desired trajectory of the rotating base are shown in Fig.8.

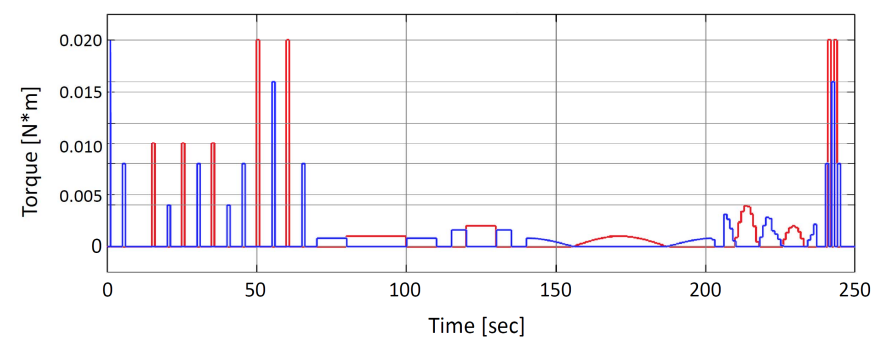

Fig. 8. Graph of the torque applied to the top (blue) and bottom (red) clutch in order the rotating base to follow the desired trajectory
It can be seen on the presented graphs, that in order to ensure the base is following the desired trajectory, the bottom MR clutch torque $T_{2}$ fluctuates from 0 up to $0.020 \mathrm{~N} \cdot \mathrm{m}$. The top MR clutch torque $T_{3}$ reaches $0.025 \mathrm{~N} \cdot \mathrm{m}$. Both clutches cannot produce negative torque due to physical limitations, so the minimum torque is $0 N \cdot m$ as shown on the graphs.

The rotation base follows the desired trajectory close enough. The graph of the simulated angle is shown in Fig.9.

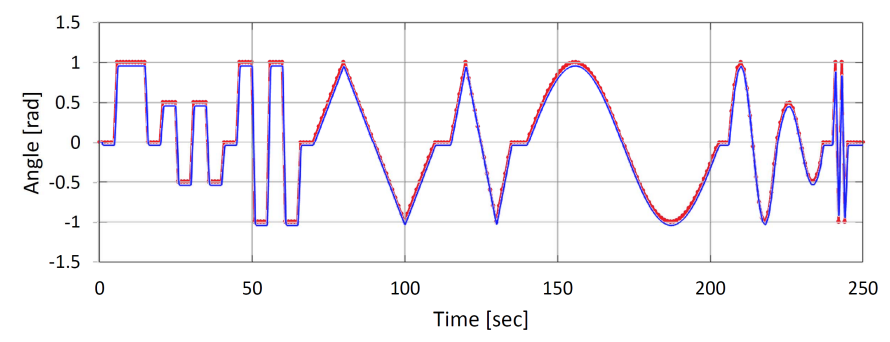

Fig. 9. Graph of the desired (red) and resulting (blue) trajectory of the rotating base.

The profile on the graph (blue) is almost identical to the profile of the desired trajectory (red) shown in Fig. 9. The error between the guiding profile and the actual angle of the base stays within +/- $0.04 \mathrm{rad}$.

\section{CONCLUSIONS}

Design and simulation results for the first joint of the prospective 5-DOF manipulator, presented in this paper, demonstrate the possibility of building the complex robot that can be actuated by a single motor at the base. The use of MR clutches in the joints of the manipulator brings power and safety to the system.

Further work is planned to model and simulate the rest of the joints in the robot. Future steps include parts manufacturing, control system development, assembling and testing the manipulator.

\section{REFERENCES}

[1] A. S. Shafer and M. R. Kermani, "Design and validation of a magnetorheological clutch for practical control applications in humanfriendly manipulation," in Robotics and Automation (ICRA), 2011 IEEE International Conference on. IEEE, 2011, pp. 4266-4271.

[2] W. Li, P. Yadmellat, and M. R. Kermani, ”Design optimization and comparison of magneto-rheological actuators," in Robotics and Automation (ICRA), 2014 IEEE International Conference on. IEEE, 2014, pp. 50505055.

[3] M. Moghani and M. R. Kermani, "Design and development of a hybrid Magneto-Rheological clutch for safe robotic applications," in Robotics and Automation (ICRA), 2016 IEEE International Conference on. IEEE, 2016, pp. 3083-3088.

[4] M. Zinn, O. Khatib, and B. Roth, "A new actuation approach for human friendly robot design," in Proc. IEEE Int. Conf. Robot. Autom., vol. 1, 2004, pp. 249-254.

[5] Dongjun Shin, I. Sardellitti, and O. Khatib, "A hybrid actuation approach for human-friendly robot design," in Robotics and Automation (ICRA), 2008 IEEE International Conference on. IEEE, 2008 pp. 1747-1752.

[6] K. Kong, J. Bae, and M. Tomizuka, "Control of rotary series elastic actuator for ideal force-mode actuation in human-robot interaction applications," Mechatronics, IEEE/ASME Transactions on, vol. 14, no. 1, 2009, pp.105-118. 
[7] A. S. Shafer and M. R. Kermani, "On the feasibility and suitability of $\mathrm{mr}$ fluid clutches in human-friendly manipulators," Mechatronics, IEEE/ASME Transactions on, vol. 16, no. 6, 2011, pp. 1073-1082.

[8] P. Fauteux, M. Lauria, B. Heintz, and F. Michaud, "Dual-differential rheological actuator for high-performance physical robotic interaction," Robotics, IEEE Transactions on, vol. 26, no. 4, 2010, pp. 607-618.

[9] S. Diller, C. Majidi, and S. Collins, "Exoskeleton walking with a lightweight , low power electroadhesive clutch and spring," in Robotics and Automation (ICRA), 2016 IEEE International Conference on. IEEE, 2016, pp. 682-689.

[10] A. S. Shafer and M. R. Kermani, "Development of high performance intrinsically safe 3-DOF robot," in Robotics and Automation (ICRA), 2014 IEEE International Conference on. IEEE, 2014, pp. 619-624.

[11] M. S. Santina, A. R. Stubberud, and G. H. Hostetter, Digital Control System Design, 2nd ed. Orlando, United States of America: Saunders College Publishing, a Harcourt Brace College Publisher, 1994. 